\title{
10. Effects of Magnetic Field on the Striated Electric Discharge Figure.
}

By Tadasi ITOH and Katuhiko YonETA.

Faculty of Science, Hokkaido Imperial University.

(Rec. Feb. 1, 1932. Comm. by T. Terada, M.I.A., Feb. 12, 1932.)

The interesting experiments of applying strong magnetic field on electric discharge figures made by C. E. Magnusson ${ }^{1)}$ led us to try the similar experiments for the striated discharge figures previously reported by one of the present authors. ${ }^{2)} \quad$ The characteristic discharge figures can be generated at low gas pressure of several $\mathrm{cm}$. under intermittent impulsive electromotive force, of which the voltage is far smaller compared with that of an ordinary electric figure due to a single impulse. This latter is a point of convenience which may be regarded as the characteristic of the present experiment. The electromagnet used was made in this laboratory, consisting of a cylindrical coil with a concentric core of soft iron of $5 \mathrm{~cm}$. diameter. Maximum available field at $1 \mathrm{~cm}$. from the end of the core was about 2,200 gauss.

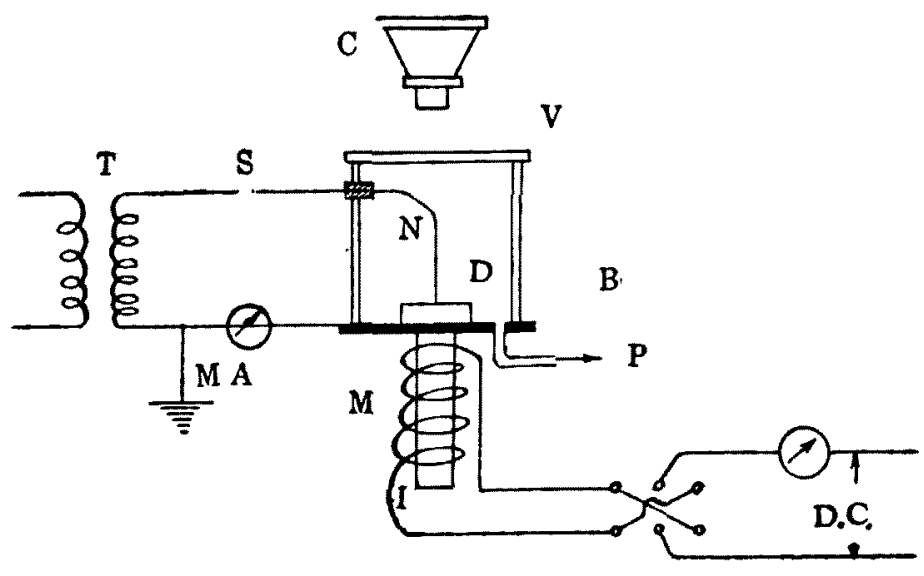

Fig. 1.
$\mathrm{T}$ : High tension transformer

$\mathrm{N}$ : Needle point electrode of brass

D: Dielectric (glass or oil)

B : Brass plate

V: Glass vessel

S: Spark gap having conical ends

C: Photographic camera

MA : Milli-ammeter

$P$ : To pressure gauge and pump

M : Electro-magnet

I: Iron core

The apparatus and the electric connection used are roughly shown in Fig. 1. First, the striated figure was generated on the dielectric, and then the magnetic field was applied. Thus, the shift of the striae, the change in size and appearance of the discharge figure due to the

1) C. E. Magnusson: A. I. E. E., Oct. 1930, p. 1834.

2) T. Itoh: Proc. 4 (1928), 16, 286; 5 (1929), 5, 9, 12; Memoirs of the Faculty of Eng., Hokkaido Imp. Univ., 1 (1928), No. 5, 237; J. Fac. Science, Hokkaido Imp. Univ., Ser. II, 1 (1930), 57; (1931), 77. 
No. 2.] Effects of Magnetic Field on the Striated Electric Discharge Figure.

applied magnetic field were observed. The amount and the direction of the striae shift depend, not only on the gas pressure and magnetic field, but in some degree also on the surface and boundary conditions of the dielectric. In most of the present observations, the striae were curved and shifted to its concave side as shown in Figs. 2 and 4.

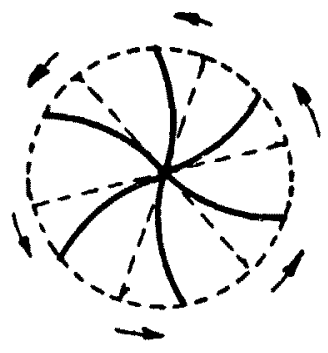

Fig. 2.

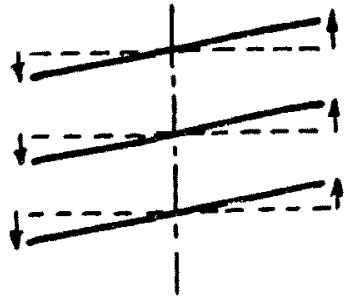

Fig. 3.

In the case of radially striated discharges, the sense of the curvature of the striae under the magnetic field is the same as in the result by Magnusson following Fleming's Right-Hand Rule for negative charges under the influences of the electric and magnetic fields.

The dark straight line separating two similar discharge figures (generated around the two needle electrodes) is rotated and curved in the fixed direction according to the sense of the applied magnetic field but not of the polarity of the electrodes. (Figs. 5, 6).

The existence of a kenetron in the discharge circuit connected for giving positive impulses gives very little effect on both the figure and the effect of the magnetic field.

If the spark gap $\mathrm{S}$ in Fig. 1 is replaced parallel to the discharge vessel and an alternating or positive electromotive force is applied, the magnetic effect on the figure is especially striking as shown in Fig. 7.

At a gas pressure of about $1 \mathrm{~cm}$. or less, a kind of luminous fog fills the space above the dielectric and around the whole needle. If the magnetic field is applied, this fog around the needle goes out, and the discharge takes place in form of sparks radiating from the needle point. These sparks are radiated in random directions and change into glow discharges in their way (Fig. 8). Each spark is thin, continuous from the needle point, and curves in the similar direction as before described. The curvature of the spark is nearly that of a simple circular arc as long as the spark gap length remains small, but inflections of the curve take place if the gap be made wider. Small inflections in the vertical directions are also observed in the latter case.

The parallel striated figure are affected similarly by the magnetic field as shown in Figs. 3 and 9.

We have investigated roughly the effect of magnetic field on the mode of rectification of the discharge current by means of $\mathrm{MA}$ in Fig. 1. The amount of rectification which is more or less in positive 
sense with regard to the needle electrode diminishes or even becomes negative after the magnetic field is applied.

Lastly, the authors express their best thanks to Prof. Terada for the interest he has taken in this work.

Fig. 4. Dielectric: Transformer oil, $1.5 \mathrm{~mm}$. depth.

Air pressure $3 \mathrm{~cm}$. Spark gap $0.015 \mathrm{~cm}$.

(a)

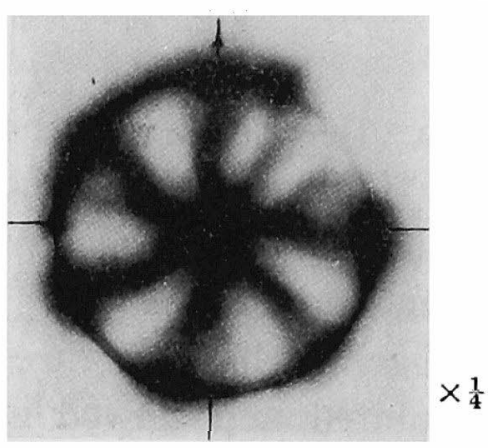

Pole S, 1,600/cm.2 (b)

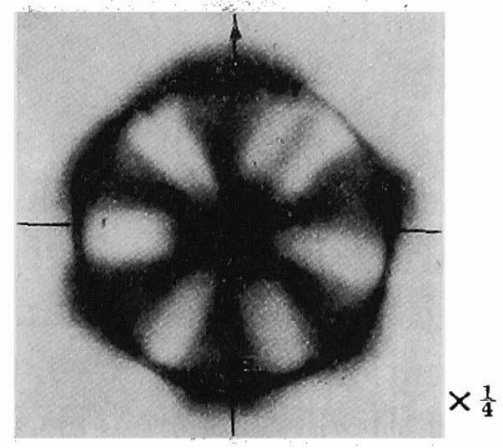

No field. (c)

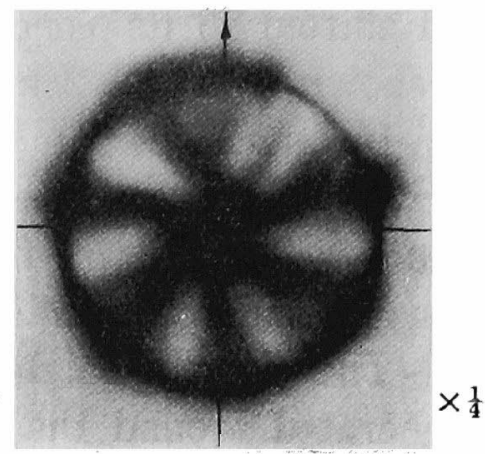

Pole N, 1,600/cm.2
Fig. 5. Glass plate, $0.17 \mathrm{~cm}$. thickness. Air pressure $4.0 \mathrm{~cm}$. Spark gap $0.05 \mathrm{~cm}$.

(a)

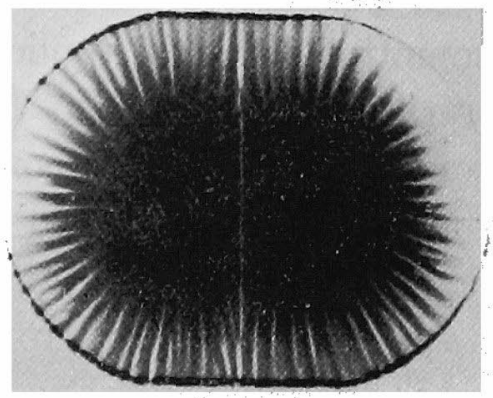

No field.

(a)

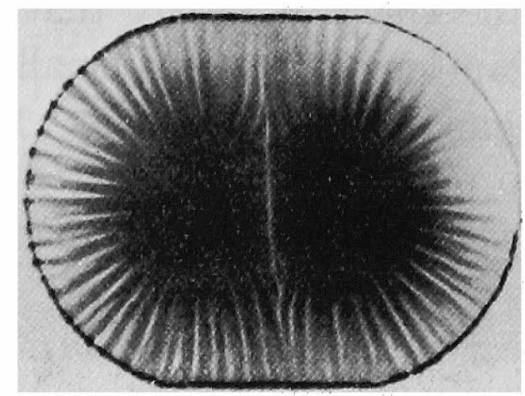

$\times \frac{1}{4}$
Fig. 6. Same dielectric as in Fig. 5. Air pressure $1.5 \mathrm{~cm}$. Spark gap $0.03 \mathrm{~mm}$.

(b)

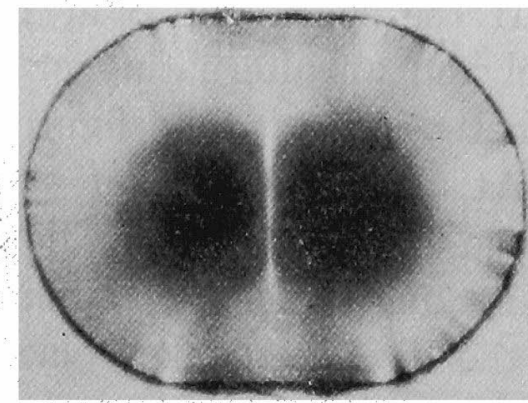

$\times \frac{1}{4}$

No field.

(b)

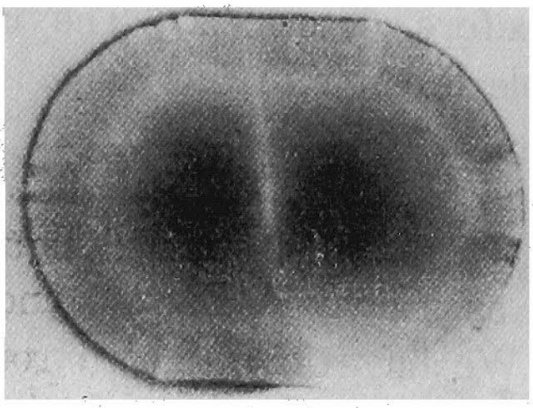

$\times \frac{1}{4}$ 
No. 2.] Effects of Magnetic Field on the Striated Electric Discharge Figure.

Fig. 7.

Glass plate $1.5 \mathrm{~cm}$. thickness. Air pressure $4.0 \mathrm{~cm}$. Spark gap $0.06 \mathrm{~cm}$.

(a)

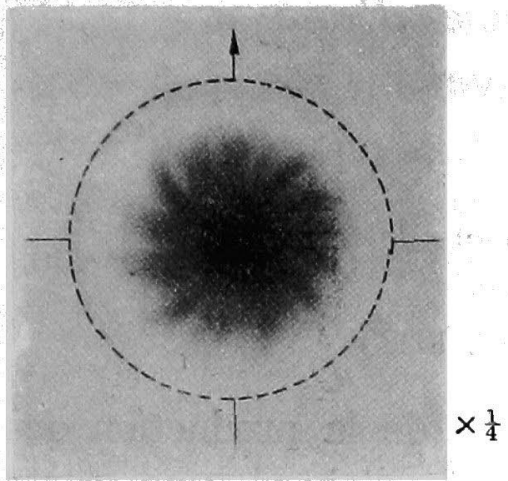

Pole S, $1,050 / \mathrm{cm}^{2}$ (b)

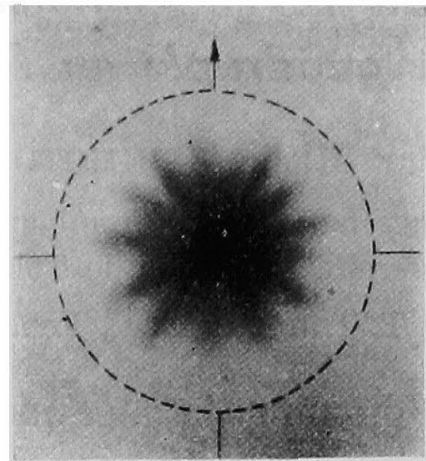

No. field.

(c)
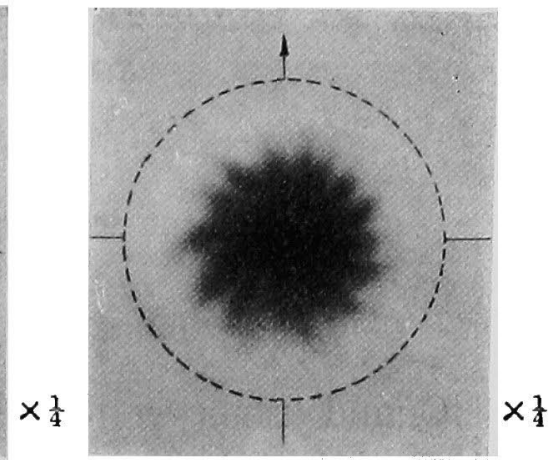

Pole N, 1,050/cm. ${ }^{2}$

Fig. 8.

Glass plate $0.17 \mathrm{~cm}$. thickness.

Air pressure $1 \mathrm{~cm}$.

Spark gap $0.3 \mathrm{~cm}$.

Pole S, 2,150/cm.2

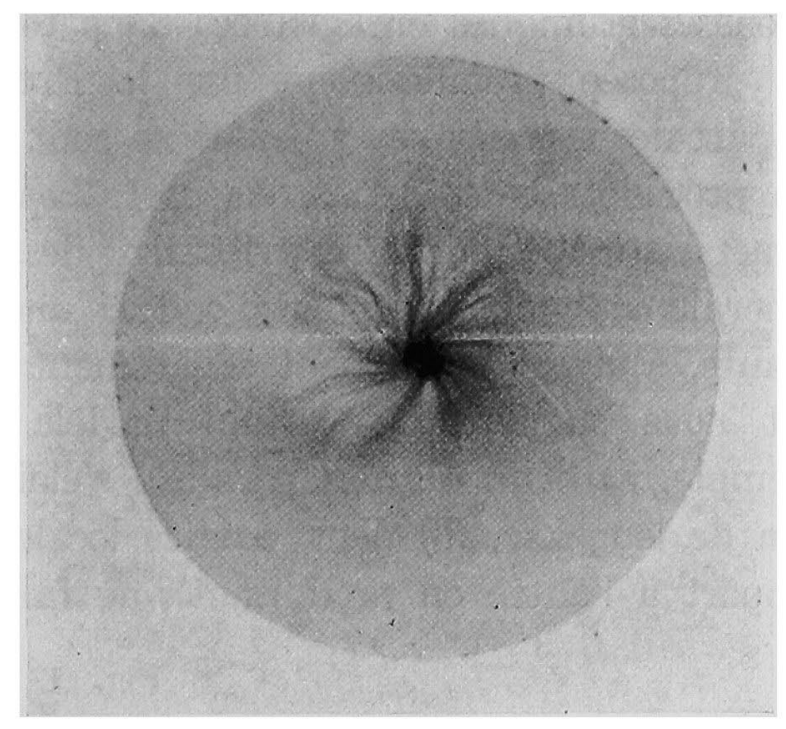

Fig. 9.

Glass plate $0.17 \mathrm{~cm}$. thickness. Air pressure $2.5 \mathrm{~cm}$. Spark gap $0.04 \mathrm{~cm}$.

(a)

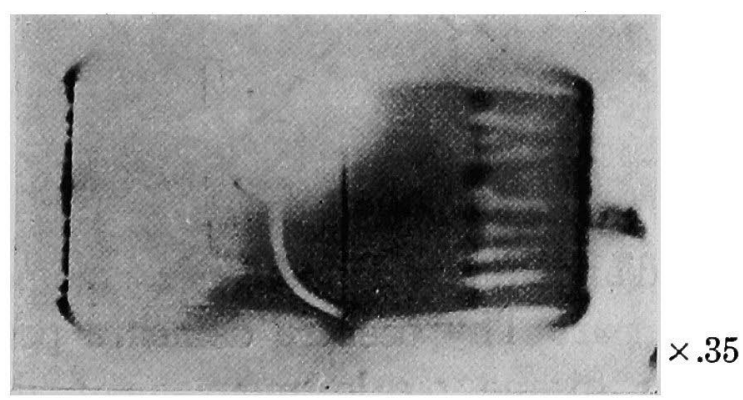

No field. (b)

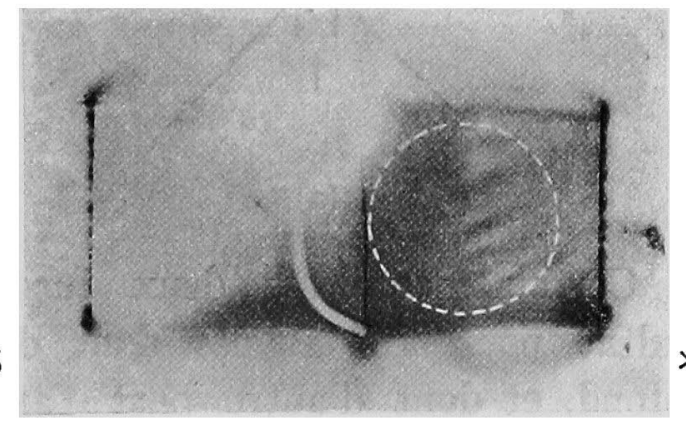

Pole N, 2,150/cm. ${ }^{2}$ 